\title{
False Alarm Reduction in Atrial Fibrillation Screening
}

\author{
Hesam Halvaei ${ }^{1}$, Emma Svennberg ${ }^{2}$, Leif Sörnmo ${ }^{1}$, Martin Stridh ${ }^{1}$ \\ ${ }^{1}$ Biomedical Signal Processing Group, Department of Biomedical Engineering, Lund University, \\ Lund, Sweden \\ ${ }^{2}$ Department of Clinical Sciences, Danderyd University Hospital, Karolinska Institutet, Stockholm, \\ Sweden
}

\begin{abstract}
Early detection of $A F$ is essential and emphasizes the significance of $A F$ screening. However, AF detection in screening ECGs, usually recorded by handheld and portable devices, is limited because of their high susceptibility to noise. In this study, the feasibility of applying a machine learning-based quality control stage, inserted between the QRS detector and AF detector blocks, is investigated with the aim to improve AF detection. A convolutional neural network was trained to classify the detections into either true or false. False detections were excluded and an updated series of QRS complexes was fed to the AF detector. The results show that the convolutional neural network-based quality control reduces the number of false alarms by $24.8 \%$ at the cost of $1.9 \%$ decrease in sensitivity compared to AF detection without any quality control.
\end{abstract}

\section{Introduction}

Atrial fibrillation (AF) is a supraventricular tachyarrhythmia originating from uncoordinated atrial contractions [1]. Detection of AF is usually based on the ECG, and the main characteristics are an irregular rhythm, absence of $P$-waves, and presence of $f$-waves. Due to the high risks associated with untreated $\mathrm{AF}$, screening is becoming increasingly common [2-5].

A large number of AF detectors have been proposed over the years, see, e.g., [6-11], that may be divided into rhythm-based AF detectors and rhythm- and morphologybased AF detectors. Rhythm-based AF detectors explore the degree of irregularity in the RR interval series and can be based on entropy measures [9], statistical measures [8], or Poincaré plot features [7]. Rhythm- and morphology-based AF detectors, in addition to rhythm irregularity information, detects atrial activity, i.e., P-waves or f-waves [10,11].

AF screening is usually performed using handheld and portable devices. These devices allow for simplified measurements at home but the recorded signals are often of lower quality compared to the conventional ECG recordings $[12,13]$. A lower signal quality influences the performance of AF detection algorithms and typically leads to a higher false alarm rate. Therefore, handling of lower signal quality is an important problem to address. In this study, we propose a signal quality control stage and investigate its influence on AF detection performance in short-term screening ECGs. This stage is a CNN that classifies the outputs of the QRS detector described in [14] into either true or false detections. A rhythm-based AF detector was used since atrial activity is difficult to quantify in screening ECGs. The significance of the CNN-based quality control is investigated on the Physionet/CinC 2017 Challenge database [15].

\section{Databases}

Two databases were used for method development and evaluation: The Physionet/CinC 2017 Challenge database (CinC17DB) [15] and the StrokeStop I database (SSIDB) [3].

SSIDB can be divided into two parts from the expert annotation perspective: signal-level and patient-level annotations. Half of the database is annotated at the signal level, indicating whether AF episodes are present in the ECG. The other half is annotated at the patient-level, indicating whether a patient has AF and does not reveal in which of the ECG recordings AF is present.

\subsection{Software-generated categorization}

In addition to the database annotations, labels generated by ECG Parser (https://cardiolund.com), a CE-marked ECG analysis software, were used. The software divides the database into the following categories: poor quality, sinus rhythm, bigeminy, trigeminy, SVES, VES, fast/slow rhythm, pause/AV block, and irregular signal, see Fig. 1. These categorization helped select training data for the CNN-based quality control stage, especially for SSIDB, where in contrast to CinC17DB, no information other than 


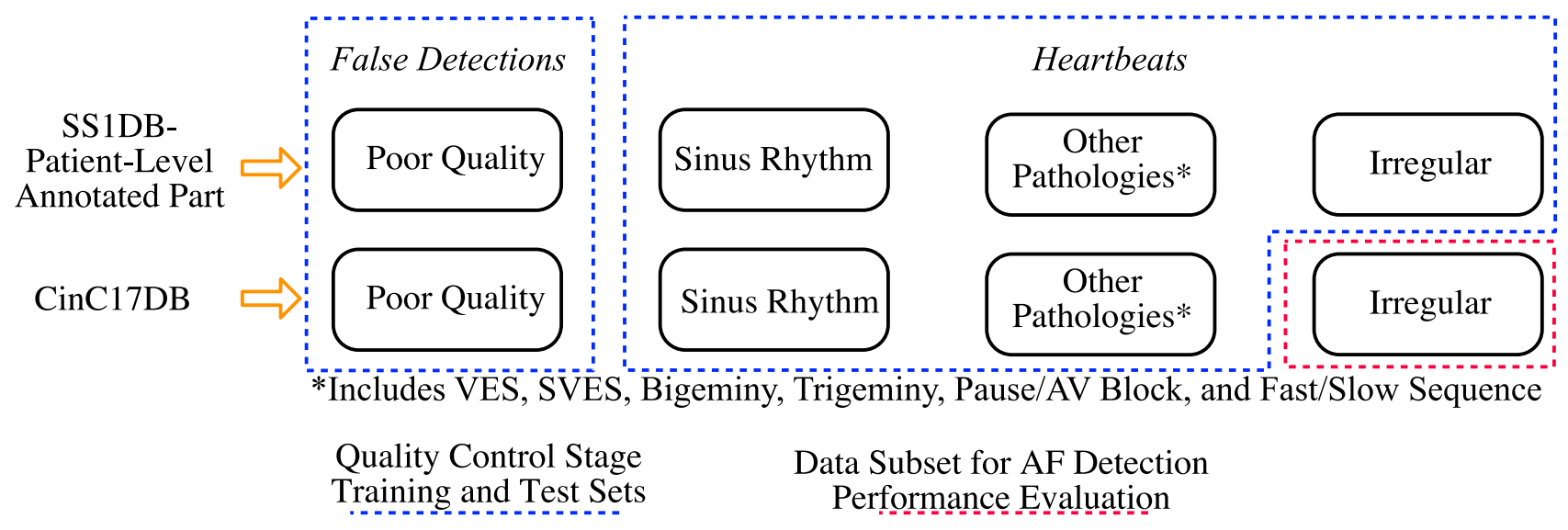

Figure 1. Software-generated categorization for SS1DB and CinC17DB

$\mathrm{AF} / \mathrm{non}-\mathrm{AF}$ was provided.

\section{Methods}

\subsection{CNN-based quality control}

Convolutional neural networks are capable of automated feature extraction, performed by convolutional layers. Convolutional layers are the main elements of the CNNs, in which multiple convolutional layers may be connected to produce feature maps. The maps are then connected to fully connected layers responsible for classification. So, by applying CNNs, feature extraction and classification, i.e., two distinct steps in traditional machine learning are grouped into one single step. The pooling layer is another element of CNNs, which summarize the produced feature maps by removing redundant information from them. This step is important as it prevents network overfitting.

In this work, we trained a CNN to distinguish the origin of detections as either of cardiac origin, i.e., heartbeats or of non-cardiac origin, i.e., false detections. Training data were composed of detections from CinC17DB and from the patient-annotated part of SS1DB. However, beat-level quality annotations were not provided with the databases. In order to handle this problem, false detections were selected from the poor quality signals, see Fig. 1. These signals were manually reviewed to ensure that no heartbeats were present. Then, all detections were labelled as false. The same approach was applied for collecting heartbeats. In this case, heartbeats were selected from a wide range of ECGs in order to include different rhythm and beat morphologies into the heartbeat category. The irregular signals in CinC17DB were saved and used exclusively for the $\mathrm{AF}$ detection performance evaluation.

\subsection{AF detection}

In this study, the low complexity AF detector [9] was used, offering good performance according to the comparison in [16]. The AF detector quantifies RR interval irregularity in a four-beat sliding time window, using a simplified sample entropy measure. Once this measure exceeds a fixed threshold $\eta$, an AF episode is detected.

\subsection{Performance evaluation}

The performance is reported for both the CNN-based quality control stage and the $\mathrm{AF}$ detector. The receiver operating characteristics (ROC) curve displays the performance of the CNN-based quality control stage in terms of sensitivity (Se) versus false positive rate (FPR) for different classification/detection thresholds; Se and FPR are defined by:

$$
\begin{gathered}
S e=\frac{T P}{T P+F N}, \\
F P R=\frac{F P}{T N+F P},
\end{gathered}
$$

where TP is the number of true positives, FP is the number of false positives, $\mathrm{TN}$ is the number of true negatives, and $\mathrm{FN}$ is the number of false negatives.

\section{Results}

In total, 1267 ECGs were selected from both databases. From these ECGs, 52194 beats were extracted, of which, 19763 were false detections. These detections were divided into a $80 \%$ training and cross-validation set and $20 \%$ test set. The training data were augmented by inverting all the detections in the training set in order to handle inverted recordings better. The ROC curve for the CinC17DB detection test set is displayed in Fig. 3. It shows that an AUC of 0.99 was obtained for CinC17DB. 


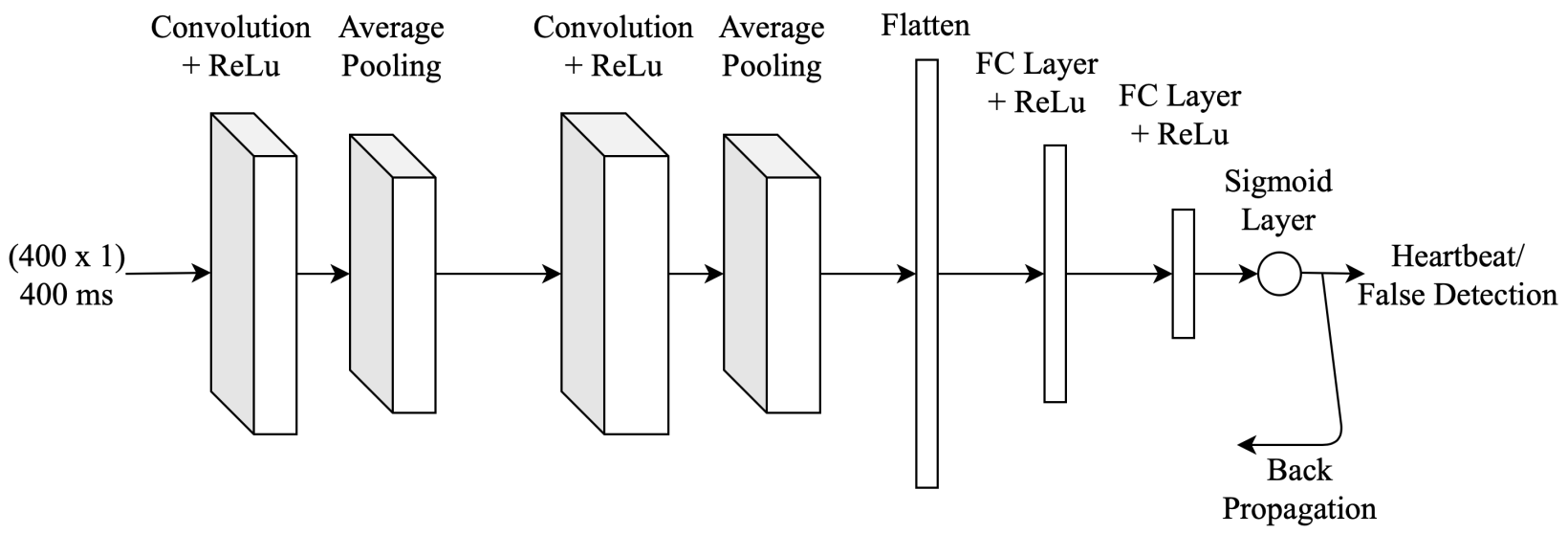

Figure 2. Structure of the proposed CNN for quality control

The trained CNN was applied to the group of irregular signals in the CinC17DB to investigate the effect of quality control on AF detection performance. The AF detection sensitivity and FPR for different AF detection thresholds are displayed in Fig. 4, with and without using the quality control stage. A high sensitivity achieved without quality control came at the expense of a high false positive rate, see Fig. 4.

By inserting the quality control stage, the false positive rate was reduced by $15.9 \%-24.8 \%$, while the sensitivity decreased marginally. For the highlighted threshold at 0.39 in Fig. 4, the CNN-based quality control stage reduced the false positive rate by $24.8 \%$ compared to AF detection alone. This improvement was achieved at the expense of $1.9 \%$ reduction in sensitivity.

\section{Discussion}

In this study, we applied a CNN to identify and exclude false QRS detections produced by the QRS detector, which deteriorate the performance of the rhythm-based AF detector. The quality control stage could have been merged with the QRS detector, the AF detector, or the whole pipeline could have been integrated into one ML-based box. However, merging would disrupt the traceability of decisions, while using the proposed pipeline, the decisions can be traced backwards. Besides, well-established methodology for QRS and AF detection does not have to be reinvented. The resulting pipeline provided a significant reduction in the false positive rate at the expense of small reduction in sensitivity.

Different QRS detectors and AF detectors could have been used. However, false detections by the QRS detector in noisy signals and numerous false AF alarms in screening databases are well-documented. The solution proposed in this work may be combined with any other modules in dif-

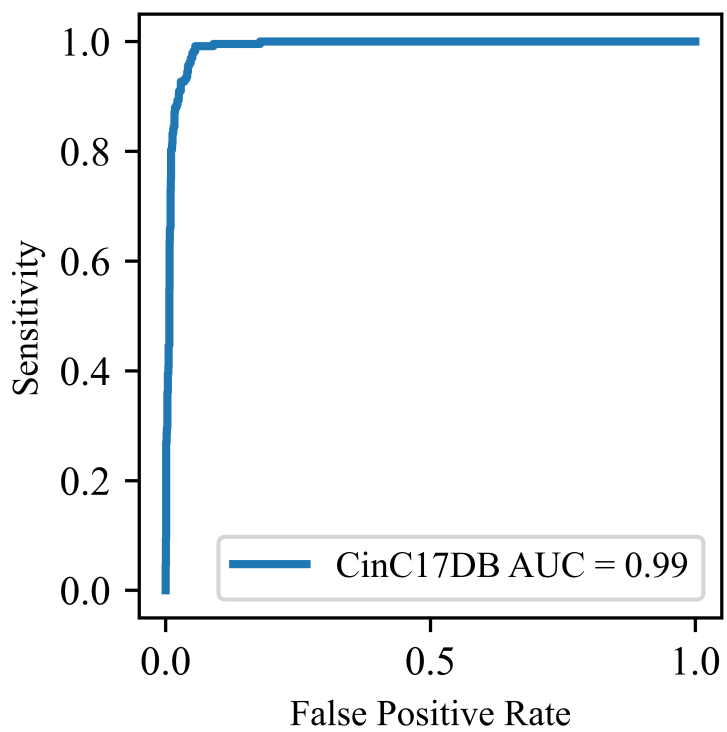

Figure 3. The ROC for CinC17DB detections test set.

ferent settings and the results in this paper represents an example of performance improvement that can be achieved.

\section{Acknowledgments}

This research was supported by the European Union's Horizon 2020 research and innovation programme under the Marie Sklodowska-Curie grant agreement No.766082 (MY-ATRIA project).

\section{References}

[1] Platonov PG, Corino VD. A clinical perspective on atrial fibrillation. In Atrial Fibrillation from an Engineering Per- 
AF Detection Performance Without Quality Control

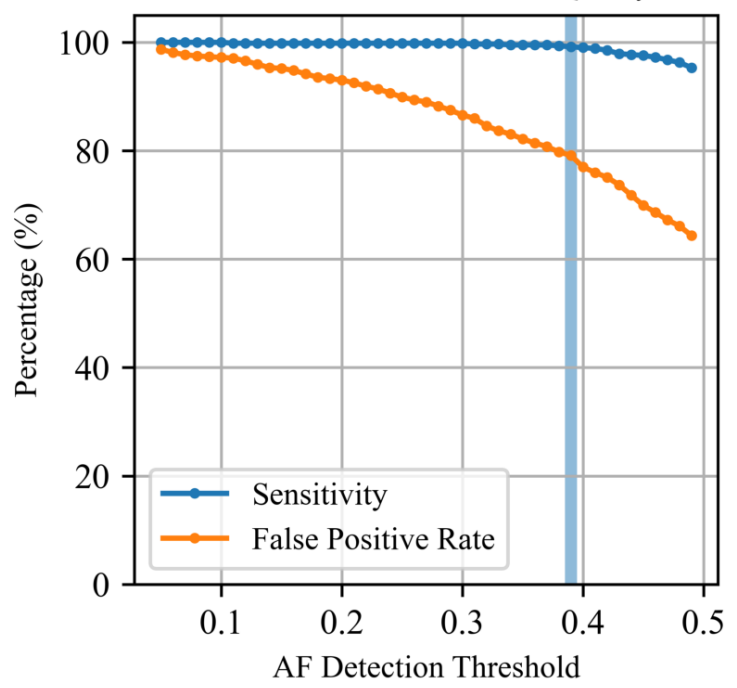

AF Detection Performance With Quality Control

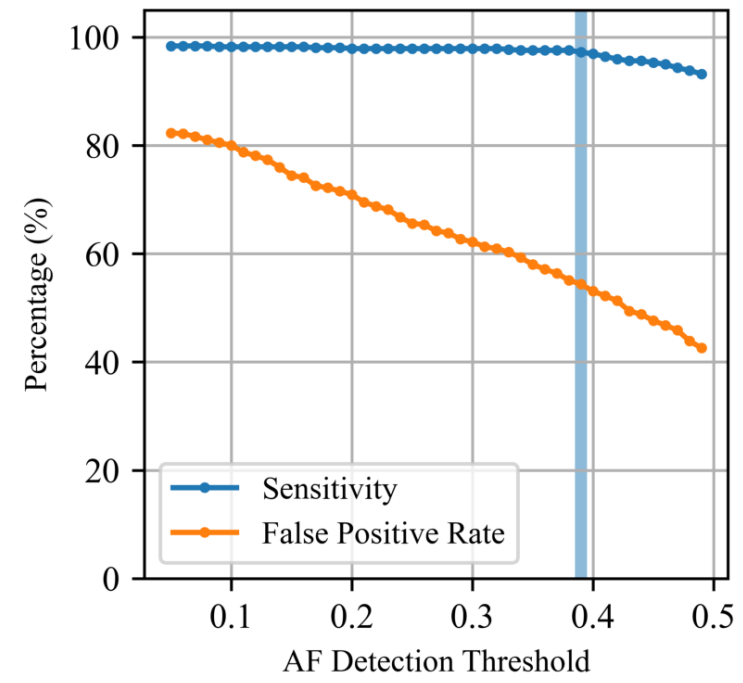

Figure 4. AF detection performance on CinC17DB irregular signals

spective (L. Sörnmo, ed). Springer, 2018; 1-24.

[2] Engdahl J, Andersson L, Mirskaya M, Rosenqvist M. Stepwise screening of atrial fibrillation in a 75-year-old population: implications for stroke prevention. Circulation 2013; 127(8):930-937.

[3] Svennberg E, Engdahl J, Al-Khalili F, Friberg L, Frykman V, Rosenqvist M. Mass screening for untreated atrial fibrillation: the strokestop study. Circulation 2015; 131(25):2176-2184.

[4] Proietti M, Mairesse GH, Goethals P, Scavee C, Vijgen J, Blankoff I, Vandekerckhove Y, Lip GY. A population screening programme for atrial fibrillation: a report from the belgian heart rhythm week screening programme. Ep Europace 2016;18(12):1779-1786.

[5] Chan Ny, Choy Cc. Screening for atrial fibrillation in 13 122 hong kong citizens with smartphone electrocardiogram. Heart 2017;103(1):24-31.

[6] Dash S, Chon K, Lu S, Raeder E. Automatic real time detection of atrial fibrillation. Annals of biomedical engineering 2009;37(9):1701-1709.

[7] Lian J, Wang L, Muessig D. A simple method to detect atrial fibrillation using rr intervals. The American journal of cardiology 2011;107(10):1494-1497.

[8] Huang C, Ye S, Chen H, Li D, He F, Tu Y. A novel method for detection of the transition between atrial fibrillation and sinus rhythm. IEEE Transactions on Biomedical Engineering 2010;58(4):1113-1119.

[9] Petrėnas A, Marozas V, Sörnmo L. Low-complexity detection of atrial fibrillation in continuous long-term monitoring. Computers in biology and medicine 2015;65:184-191.

[10] Ródenas J, García M, Alcaraz R, Rieta JJ. Wavelet entropy automatically detects episodes of atrial fibrillation from single-lead electrocardiograms. Entropy 2015;17(9):6179-
6199.

[11] Ladavich S, Ghoraani B. Rate-independent detection of atrial fibrillation by statistical modeling of atrial activity. Biomedical Signal Processing and Control 2015;18:274281.

[12] Svennberg E, Stridh M, Engdahl J, Al-Khalili F, Friberg L, Frykman V, Rosenqvist M. Safe automatic one-lead electrocardiogram analysis in screening for atrial fibrillation. Ep Europace 2017;19(9):1449-1453.

[13] Freedman B, Camm J, Calkins H, Healey JS, Rosenqvist M, Wang J, Albert CM, Anderson CS, Antoniou S, Benjamin EJ, et al. Screening for atrial fibrillation: a report of the af-screen international collaboration. Circulation 2017; 135(19):1851-1867.

[14] Lagerholm M, Peterson C, Braccini G, Edenbrandt L, Sornmo L. Clustering ecg complexes using hermite functions and self-organizing maps. IEEE Transactions on Biomedical Engineering 2000;47(7):838-848.

[15] Clifford GD, Liu C, Moody B, Li-wei HL, Silva I, Li Q, Johnson A, Mark RG. Af classification from a short single lead ecg recording: the physionet/computing in cardiology challenge 2017. In 2017 Computing in Cardiology (CinC). IEEE, 2017; 1-4.

[16] Sörnmo L, Petrènas A, Marozas V. Detection of atrial fibrillation. In Atrial Fibrillation from an Engineering Perspective. Springer, 2018; 73-135.

Address for correspondence:

Hesam Halvaei

Lund University, Department of Biomedical Engineering, Box 118, 22100, Lund, Sweden

hesam.halvaei@bme.lth.se 